\title{
Descending Thoracic Aorta Stenosis Treated by Percutaneous Transluminal Angioplasty and Stenting with Coaxial Sheath Introduction Technique in a Small Child
}

\author{
Binh Ho Anh' \\ Duy Le Van' \\ Khoa Phan Anh' \\ Ngoc Nguyen Thi Bich' \\ Chau Nguyen Ngoc Minh ${ }^{2}$ \\ Khanh Vo Van' \\ 'Department of Emergency and \\ Interventional Cardiology, Hue Central \\ Hospital, Hue City, Vietnam; ${ }^{2}$ Pediatric \\ Center, Hue Central Hospital, Hue City, \\ Vietnam
}

\begin{abstract}
A 10-year-old girl (23 kg) having a medical history of uncontrolled hypertension was presented to our hospital because of acute left heart failure. Transthoracic echocardiography showed stenosis of descending thoracic aorta with a maximum transstenotic pressure gradient of $50 \mathrm{mmHg}$ and severe left ventricular systolic dysfunction with an ejection fraction of $20 \%$. She was diagnosed with Takayasu arteritis with a long severe stenosis of segment III of the thoracic aorta. The procedure of percutaneous transluminal angioplasty was performed and helped to reduce the pressure gradient significantly. After a 6-month follow-up, the left ventricular function was unimproved. Hence, aortic angiography was done and revealed the descending thoracic aorta restenosis with a pressure gradient of $46 \mathrm{mmHg}$. Despite the difficulties of small vascular access and the disease severity, this patient was intervened by cover stent without any complications. The trans-stenotic pressure gradient decreased remarkably to $5 \mathrm{mmHg}$. The stent implantation should be considered in the severe stenosis of descending thoracic aorta because of its benefit and safety.
\end{abstract}

Keywords: aorta, stenosis, stent, children

\section{Introduction}

Aortic coarctation which is defined as the stenosis between the distal juxta-ductal thoracic aorta and the left subclavian artery happens in 1 per 3000-4000 live births and may be related to other innate heart defects like bicuspid aortic valve, congenital aortic stenosis, persistent ductus arteriosus, or ventricular septal defects. It accounts for $5-8 \%$ of total congenital heart disease. ${ }^{1}$

Atypical coarctation (also named middle aortic syndrome), which refers to the narrowing lesions at the aortic segments II-IV, is considered as hypoplastic types classified as congenital defects. These hypoplasias make up about $0.5-1.5 \%$ of aortic coarctation cases. ${ }^{2}$ In 1963 , Sen et al first described clearly the middle aortic syndrome as inflammatory subisthmic aortic stenosis, ${ }^{3}$ which commonly results from other inflammatory diseases such as Takayasu's arteritis, aortoarteritis, and granulomatous arteritis. ${ }^{4}$

Percutaneous transluminal angioplasty was first performed in the 1980s to approach the aortic coarctation and in the 1990s. Stent implantation was widely accepted as an
Correspondence: Duy Le Van Department of Emergency and Interventional Cardiology, Hue Central Hospital, I6 Le Loi Street, Hue City, Vietnam

Email Ivnduy@gmail.com 
alternative, which has become a treatment of choice nowadays for children and adults in many institutions. ${ }^{5}$

We present a 10-year-old girl with severe descending thoracic stenosis that was successfully undertaken percutaneous transluminal angioplasty and stent implantation at our cardiovascular center.

\section{Case Report}

A 10-year-old girl (23kg of weight and $125 \mathrm{~cm}$ of height) who had a medical history of uncontrolled hypertension despite optimal antihypertensive therapy and chronic dyspnea was transferred to Hue Central Hospital for severe heart failure.

On physical examination, resting blood pressure was $150 / 90 \mathrm{mmHg}$, and femoral arteries pulses were weak on both sides. There was a severe left ventricular dysfunction with an ejection fraction of $20 \%$ and Pro-BNP of $18,179 \mathrm{pg} /$ $\mathrm{mL}$. Chest radiography indicated an elevated cardiothoracic ratio of more than 55\% and ECG showed sinus tachycardia. Transthoracic echocardiography confirmed the diagnosis of severe descending thoracic aorta stenosis with a pressure gradient of $50 \mathrm{mmHg}$. After an optimal medication treatment with digoxin, diuretics, and angiotensin-converting enzyme inhibitor, angiography was performed for precise information about the location and dimensions of the aortic stenosis. There was a long lesion of the whole segment III of the thoracic aorta (Figure 1). The procedure of percutaneous transluminal angioplasty was performed with Tyshask 8 and $10 \mathrm{~mm}$ (Figure 2). During the catheterization procedure, the

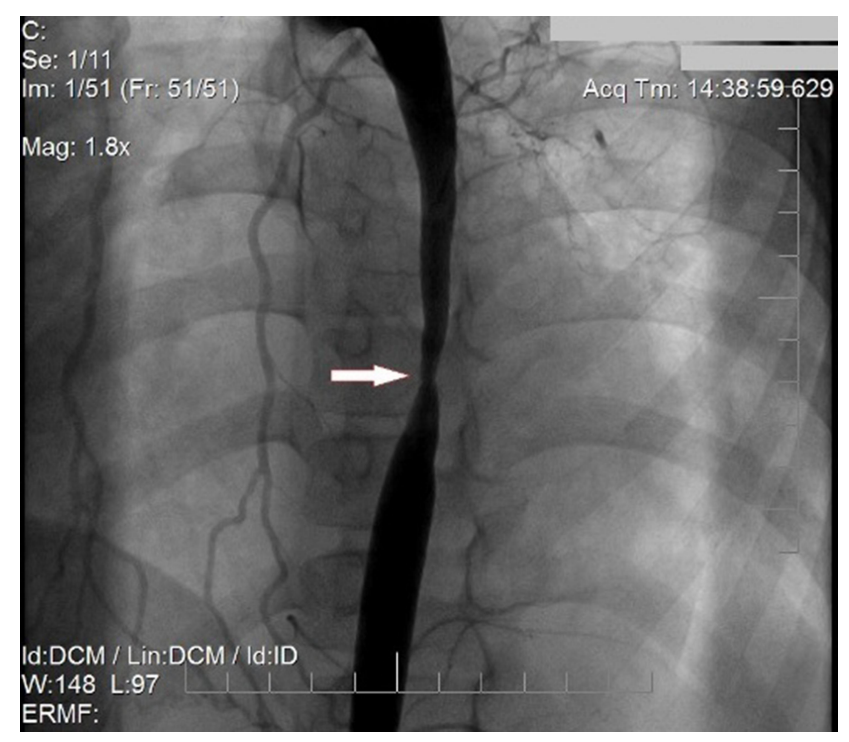

Figure I Aortic angiography demonstrates the location and dimensions of the aortic stenosis. trans-stenotic gradient before and after the procedure was 45 and $8 \mathrm{mmHg}$ respectively. After 24 hours, postprocedural echocardiography showed partial improvement of left ventricular function.

After a 6-month follow-up, she gained $2 \mathrm{~kg}$, but the dyspnea remained, and the rest blood pressure was still around $140 / 80 \mathrm{mmHg}$. This patient was readmitted to the hospital for transthoracic echocardiography, which showed a trans-stenotic gradient increased to $40 \mathrm{mmHg}$. The severe descending aorta restenosis was confirmed by angiography with the proximal and distal lumen diameter of 13.03 and $9.52 \mathrm{~mm}$, correspondingly. The narrowest point was estimated at $2.33 \mathrm{~mm}$. The length of aortic stenosis was $96 \mathrm{~mm}$, and the catheter-measured gradient was $46 \mathrm{mmHg}$. The left and right common iliac arteries were 3.86 and $3.69 \mathrm{~mm}$, respectively.

Given the severe heart failure with feasible vascular access, aortic covered-stent implantation was indicated with the vascular access of the right common femoral artery (using sheath 6Fr and 2 Perclose ProGlide) and right brachial artery (using sheath $5 \mathrm{Fr}$ ).

The procedure was performed under general anesthesia. The patient was given $100 \mathrm{UI} / \mathrm{kg}$ Heparin during the procedure. Aortic angiography confirmed the stenosis by pigtail $5 \mathrm{Fr}$ via brachial artery, then a back-up SuperStiff $0.035 " 260 \mathrm{~cm}$ long guide-wire was introduced into the ascending aorta.

A 10Fr Long Sheath (Cook Device) was initially introduced by the right common femoral approach over the stiff guide-wire, but it was impossible to be inserted due to its large size and weakness. Hence, it was strengthened by an 8Fr Amplatzer Delivery inside, and the whole delivery system, including an inner 8Fr Amplatzer Delivery covered by a 10 Fr Cook Outer Sheath, was successfully inserted into the descending aorta without any difficulties (Figure 3). Two $12 \times 59 \mathrm{~mm}$ Balloon-Expandable Covered Stents (Begraft) were completely deployed to the distal and proximal parts of the lesion at 12 and 14 atm, respectively, then a Tyshask $14 \mathrm{~mm}$ was used to optimize stent expansion (Figures 4 and 5). Ultimately, the trans-stenotic gradient measured by catheterization decreased considerably to approximately $5 \mathrm{mmHg}$.

Two weeks after the stent implantation, the patient experienced considerable improvement. The dyspnea was reduced significantly, femoral pulses were felt clearly on both sides and blood pressure was around 100/60 mmHg. On Doppler abdominal ultrasonography, there were no significant post-procedural stenosis at the 


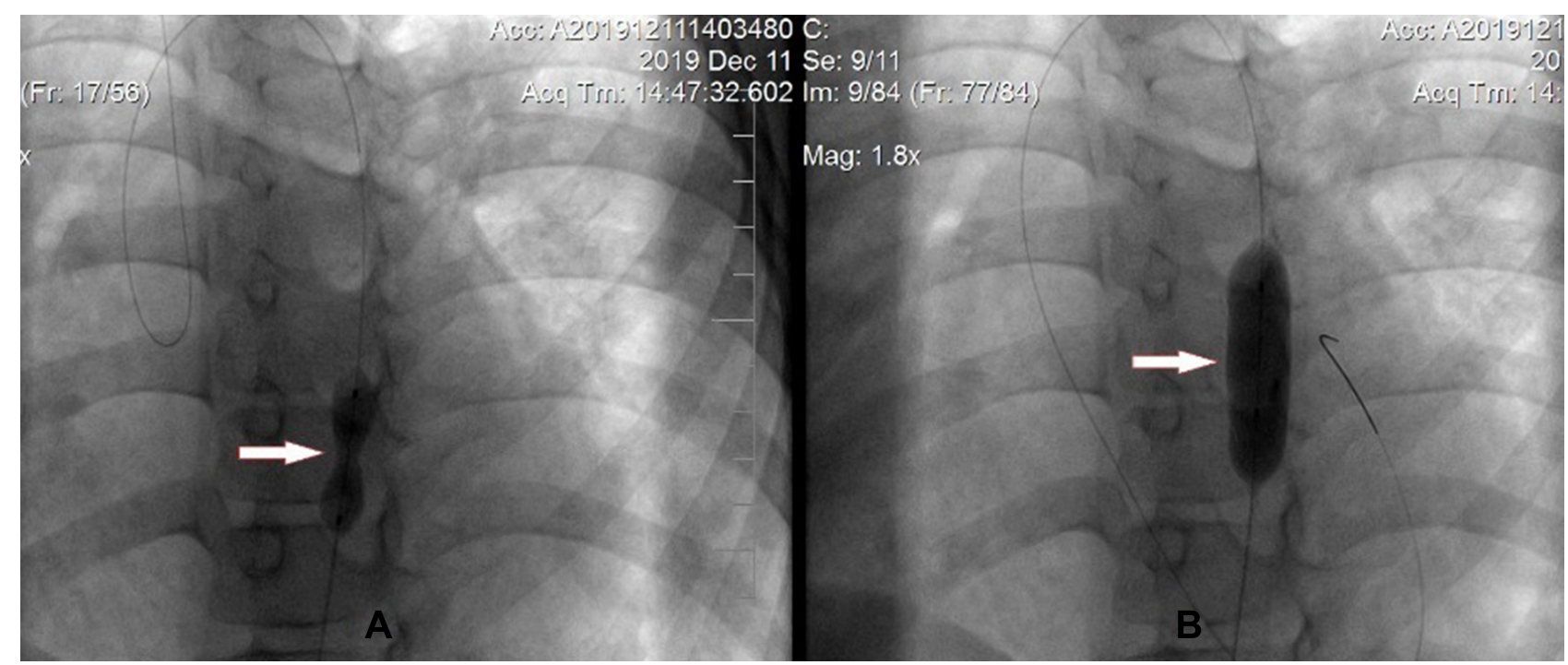

Figure 2 Percutaneous transluminal angioplasty was performed with Tyshask 8 (A) and $10 \mathrm{~mm}(\mathbf{B})$.

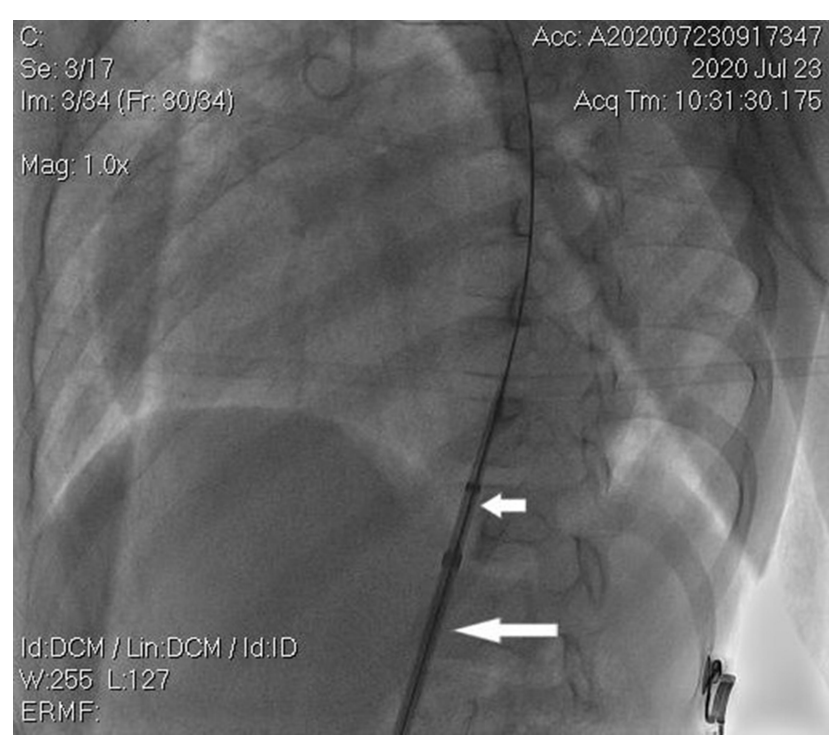

Figure 3 An inner 8Fr Amplatzer Delivery (short arrow) covered by a 10Fr Cook Outer Sheath (long arrow) was inserted into the descending aorta.

femoral puncture site and its patency was well maintained. No complications of vascular access were found. Left ventricular function improved remarkably with a rise of $25 \%$ in ejection fraction and trans-stenotic gradient was around $8 \mathrm{mmHg}$ measured by transthoracic echocardiogram. The diameter of the narrowing segment returned to the normal range (more than $90 \%$ of the normal descending aortic segment) (Figure 6).

Periodic follow-ups and echocardiogram every 3-6 months are needed to detect significant restenosis for a timely intervention.

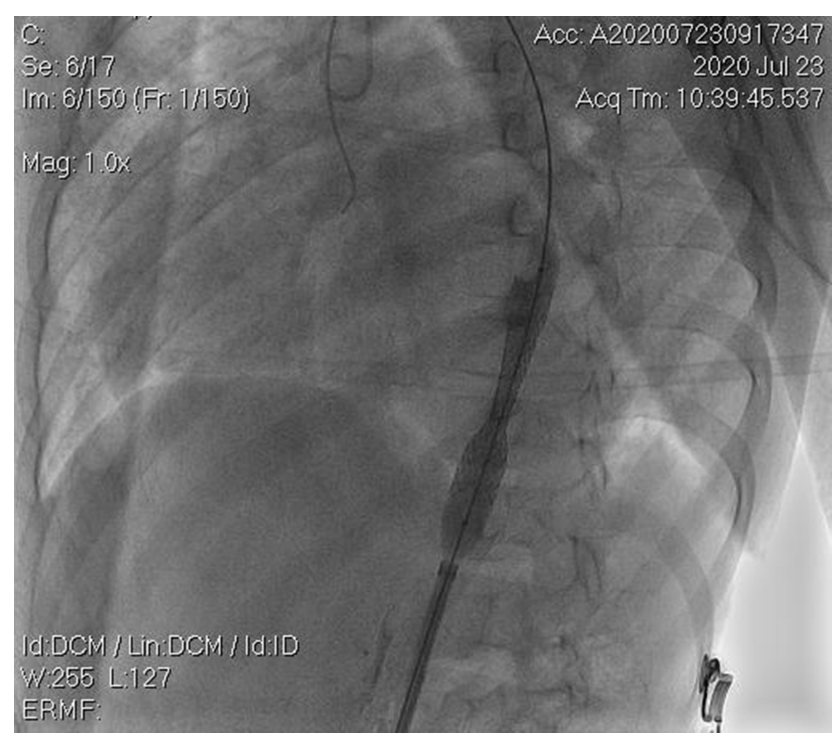

Figure 4 The first $12 \times 59 \mathrm{~mm}$ cover stent at the distal of the descending thoracic aorta.

\section{Discussion}

Given the clinical manifestations of hypertension and bilateral diminished femoral pulses, the diagnosis of Takayasu arteritis was considered on this patient and then confirmed on imaging. Angiography illustrated an atypical coarctation which was severe stenosis of segment III of the aorta. Besides the optimal pharmacological treatment, the intervention strategy should be well considered to improve the clinical condition of this patient.

Stent implantation was indicated in native and recurrent aortic coarctation according to current American 


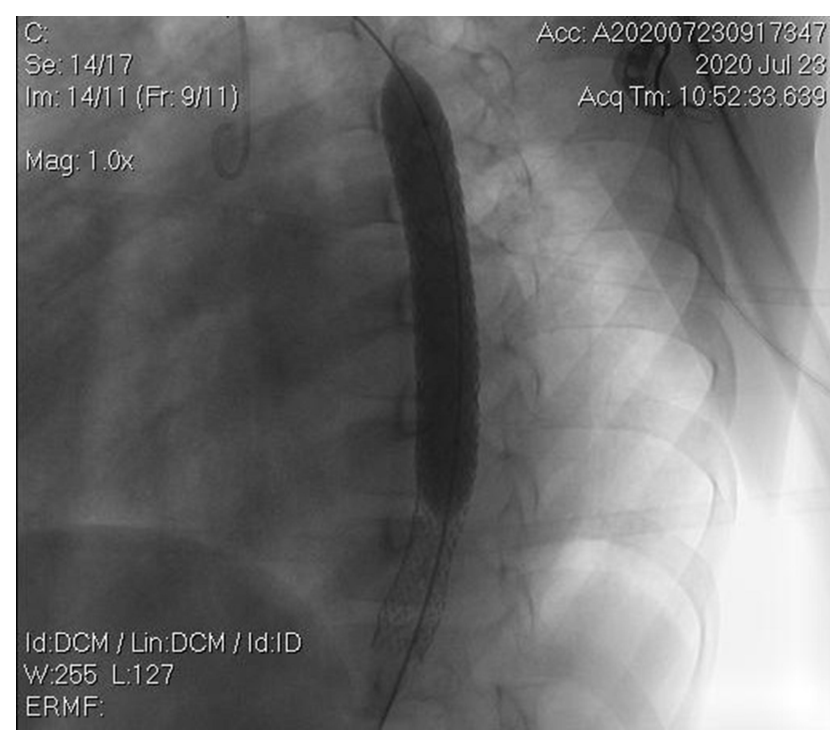

Figure 5 The second $12 \times 59 \mathrm{~mm}$ cover stent at the proximal of the descending thoracic aorta.

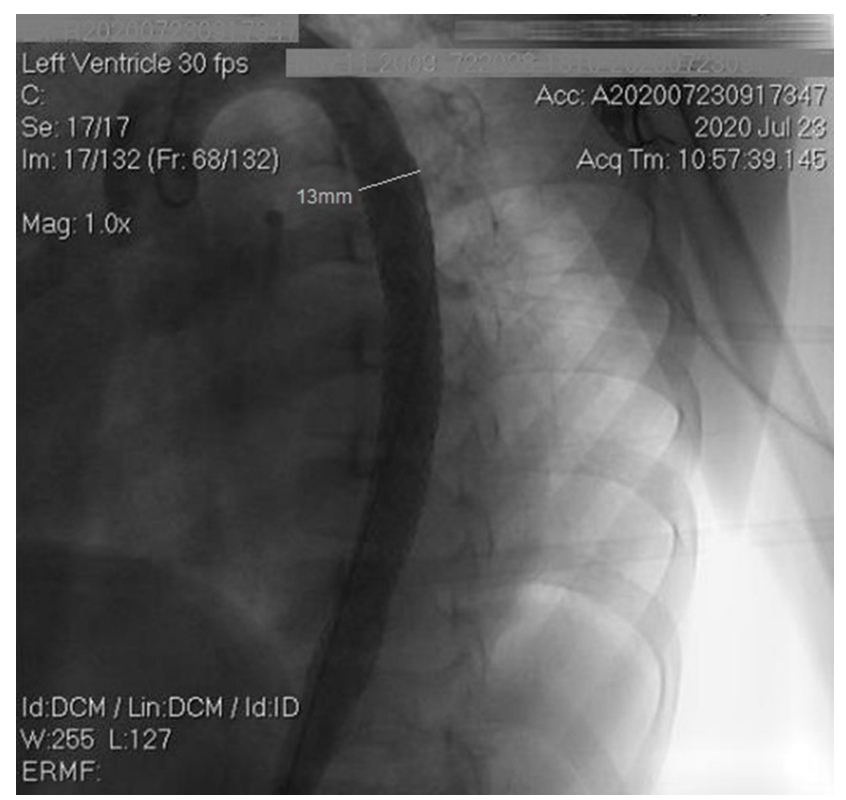

Figure 6 The ultimate result with the diameter of the narrowing segment returned to the normal range.

Heart Association guidelines. ${ }^{6}$ Hence, the choice for treatment methods, including surgical, balloon angioplasty, and stent implantation, still sparks controversy. For under-25kilogram patients with late-onset coarctation (ie, over four years old), either percutaneous transluminal angioplasty or surgery can be indicated following the Congenital Cardiovascular Interventional Study Consortium experience. ${ }^{7}$ For older and over-25-kilogram patients, the treatment options comprise balloon angioplasty and stent replacement which becomes more favorable than primary surgical treatment. ${ }^{8}$ In the situation of severe heart failure and small vessels of our patient, we hoped to decrease the trans-stenotic gradient and improve left ventricular function by percutaneous transluminal angioplasty. Therefore, the patient was partially recovered.

After 6 months, this patient was better and reached $25 \mathrm{~kg}$ of weight. The treatment of stent replacement was decided. Given the longitudinal dimension and the diameters of the injury, we chose the $12-14 \mathrm{~mm}$ covered stents and the introducer of over 9Fr diameter. However, one prominent point to emphasize is that the $10 \mathrm{Fr}$ long sheath provided by Cook Medical was the only available device in our country. The $10 \mathrm{Fr}$ sheath is equal to $3.34 \mathrm{~mm}$, smaller than the diameter of the patient's femoral arteries (3.69 and $3.86 \mathrm{~mm}$ ). After many thorough discussions of our staff including interventionists and thoracic operators, we made a very difficult decision to perform the procedure for this patient due to her severe illness. When considering the arterial access and dimensions of the stenotic lesion, we decided to use two balloon-expandable Covered Stents (Begraft). Two vascular closure devices (Perclose ProGlide) were used, and the puncture position was higher than usual to advance the $10 \mathrm{Fr}$ Long Sheath in a relatively small vascular access (Figures 7 and 8).

Because the 10Fr Long sheath was quite soft and relatively large compared to the vessel size, it was hard to be introduced to the femoral artery. This coaxial sheath introduction technique was exceptionally used after careful consideration of the whole team. An $8 \mathrm{~F}$ Amplatzer Delivery was inserted to strengthen inside the $10 \mathrm{Fr}$ Cook Outer Sheath. The whole delivery system, including an inner $8 \mathrm{~F}$ Amplatzer Delivery covered by a $10 \mathrm{Fr}$ Cook Outer Sheath, was advanced to approach the abdominal aorta. Given the length of the lesion, the narrowest point, and reference diameter, we decided that two $12 \times 59 \mathrm{~mm}$ Balloon-Expandable Covered Stents (Begraft) were optimal options. With normal pressure, the stent can obtain a diameter of $12-14 \mathrm{~mm}$ and can reach $20 \mathrm{~mm}$ after the post-dilation with a larger balloon.

In adolescents and adults, stents are recommended by most experts. A successful stent implantation procedure is defined that the residual gradient becomes less than $10 \mathrm{mmHg}$, and the narrow segment diameter improves to more than $90 \%$ of the normal adjacent aortic arch vessel. Compared to only balloon dilatation that causes persistent gradient, stent implantation is 

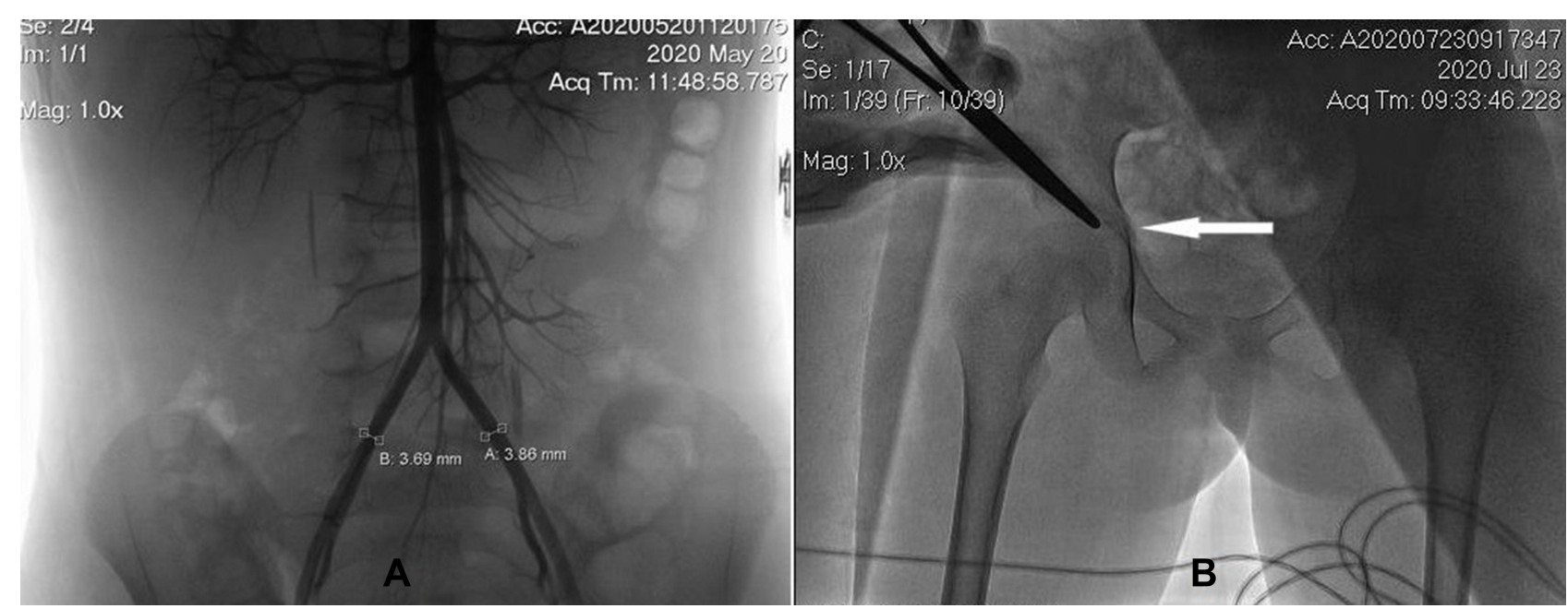

Figure 7 The vascular access (A) and puncture position (B).

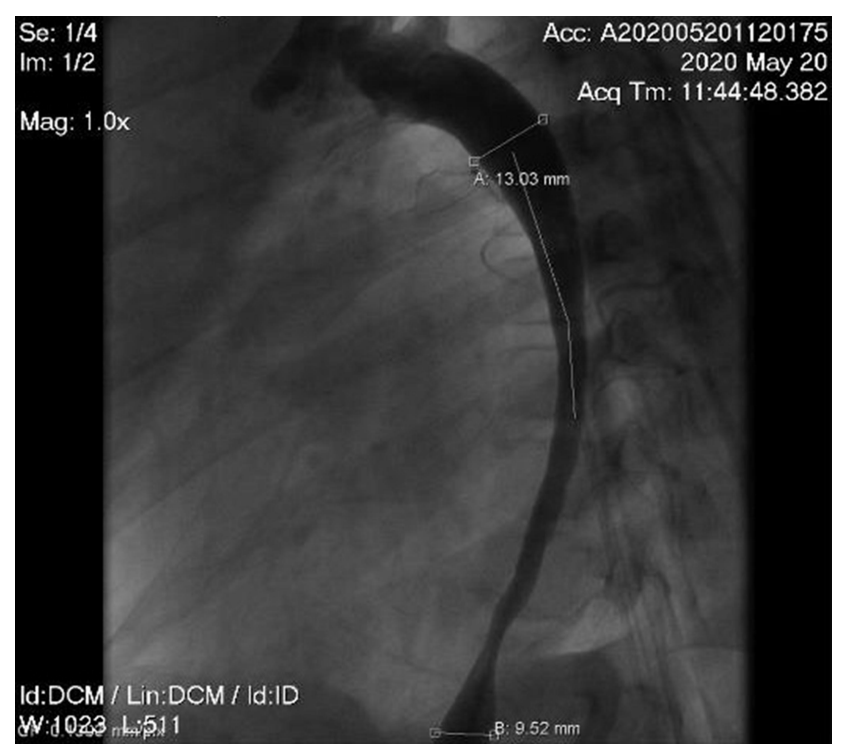

Figure 8 Aortic angiography after 6 months undergoing percutaneous transluminal angioplasty.

generally successful in more than $90 \%$ of cases without residual gradient. ${ }^{5}$ In case of the stent is implanted before puberty, the restenosis is likely to happen for many reasons such as remodeling of the coarctation lesion, development of neointimal proliferation, stent fracture or recoil, and the discrepancy between the implanted stent and the gradually increasing aortic caliber due to physical growth. These problems can be feasibly addressed by stent re-dilatation with relative safety and substantial effect. ${ }^{9}$

In this patient, a close follow-up with an echocardiogram is needed to detect significant restenosis. Therefore, the re-expansion of stents can be indicated promptly.

\section{Conclusion}

Descending thoracic stenosis, which is a popular congenital disease in children and adolescents, can result in many serious complications. The appropriate therapeutic option for adolescents with limited age and weight $(25 \mathrm{~kg})$ is challenging. The vascular closure device should be considered to replace the surgical cutdown of the femoral artery in the condition of small arterial access. Percutaneous transluminal angioplasty and stenting implantation are suitable alternatives and should be considered thanks to its efficacy and safety.

\section{Ethical Approval}

The need for ethics approval for this case report was waived.

\section{Consent for Publication}

For the publication of this case report, written informed consent was obtained from the patient's parents.

\section{Acknowledgments}

The authors thank the Department of Emergency and Interventional Cardiology of Hue Central Hospital and all the medical staff responsible for the care the patient discussed here. We appreciate the $\mathrm{HCH}$ Publication Support Team for their valuable assistance.

\section{Funding}

This work did not receive any funding. 


\section{Disclosure}

The authors report no other conflicts of interest in this work.

\section{References}

1. Murphy D. Coarctation of the aorta. In: Moodie D, editor. Congenital Heart Disease: From Infancy to Adulthood. Minneapolis: CardioText Publishing House; 2014:45-64.

2. Mickley V, Fleiter T. Coarctations of descending and abdominal aorta: long-term results of surgical therapy. J Vasc Surg. 1998;28 (2):206-214. doi:10.1016/S0741-5214(98)70156-9

3. Sen PK, Kinare SG, Engineer SD, Parulkar GB. The middle aortic syndrome. Br Heart J. 1963;25(5):610-618. doi:10.1136/hrt.25.5.610

4. Watson NA, Chalmers N, Naqvi N. Supradiaphragmatic middle aorta syndrome-MR and angiographic imaging. $B r \quad J$ Radiol. 1998;71 (842):213-216. doi:10.1259/bjr.71.842.9579186

5. Forbes TJ, Garekar S, Amin Z, et al. Procedural results and acute complications in stenting native and recurrent coarctation of the aorta in patients over 4 years of age: a multi-institutional study. Catheter Cardiovasc Interv. 2007;70(2):276-285. doi:10.1002/ccd.21164
6. Feltes TF, Bacha E, Beekman RH 3rd, et al. Indications for cardiac catheterization and intervention in pediatric cardiac disease: a scientific statement from the American Heart Association. Circulation. 2011;123(22):2607-2652. doi:10.1161/CIR.0b0 13e31821b1f10

7. Forbes TJ, Kim DW, Du W, et al. Comparison of surgical, stent, and balloon angioplasty treatment of native coarctation of the aorta: an observational study by the CCISC (Congenital Cardiovascular Interventional Study Consortium). J Am Coll Cardiol. 2011;58 (25):2664-2674. doi:10.1016/j.jacc.2011.08.053

8. Forbes TJ, Kobayashi D. Stenting coarctation of the aorta indications, techniques, and outcomes in treating native and recurrent CoA. Cardiac Interv Today. 2013;38-44.

9. Rao PS. Coarctation of the aorta. Curr Cardiol Rep. 2005;7 (6):425-434. doi:10.1007/s11886-005-0060-0

\section{Publish your work in this journal}

The International Medical Case Reports Journal is an international, peer-reviewed open-access journal publishing original case reports from all medical specialties. Previously unpublished medical posters are also accepted relating to any area of clinical or preclinical science. Submissions should not normally exceed 2,000 words or 4 published pages including figures, diagrams and references. The manuscript management system is completely online and includes a very quick and fair peer-review system, which is all easy to use. Visit http://www.dovepress.com/testimonials.php to read real quotes from published authors. 